\title{
Validation of a HPLC/FLD Method for Quantification of Tocotrienols in Human Plasma
}

\author{
Hui-Ling Che, ${ }^{1,2}$ Doryn Meam-Yee Tan, ${ }^{1,3}$ Puvaneswari Meganathan, ${ }^{1,4}$ Yee-Lin Gan, \\ Ghazali Abdul Razak, ${ }^{1}$ and Ju-Yen Fu ${ }^{1}$ \\ ${ }^{1}$ Malaysian Palm Oil Board (MPOB), No. 6, Persiaran Institusi, Bandar Baru Bangi, 43000 Kajang, Selangor Darul Ehsan, Malaysia \\ ${ }^{2}$ Department of Molecular Medicine, Faculty of Medicine, University of Malaya, 50603 Kuala Lumpur, Malaysia \\ ${ }^{3}$ International Medical University, No. 126, Jalan 19/155B, Bukit Jalil, 57000 Kuala Lumpur, Malaysia \\ ${ }^{4}$ Department of Surgery, Faculty of Medicine, University of Malaya, 50603 Kuala Lumpur, Malaysia \\ ${ }^{5}$ Department of Bioprocess Technology, Faculty of Biotechnology and Biomolecular Sciences, Universiti Putra Malaysia, \\ 43400 Serdang, Selangor, Malaysia
}

Correspondence should be addressed to Ju-Yen Fu; fujuyen@gmail.com

Received 29 July 2015; Revised 23 September 2015; Accepted 4 October 2015

Academic Editor: David M. Lubman

Copyright (C) 2015 Hui-Ling Che et al. This is an open access article distributed under the Creative Commons Attribution License, which permits unrestricted use, distribution, and reproduction in any medium, provided the original work is properly cited.

\begin{abstract}
Quantification of tocotrienols in human plasma is critical when the attention towards tocotrienols on its distinctive properties is arising. We aim to develop a simple and practical normal-phase high performance liquid chromatography method to quantify the amount of four tocotrienol homologues in human plasma. Using both the external and internal standards, tocotrienol homologues were quantified via a normal-phase high performance liquid chromatography with fluorescence detector maintained at the excitation wavelength of $295 \mathrm{~nm}$ and the emission wavelength of $325 \mathrm{~nm}$. The four tocotrienol homologues were well separated within 30 minutes. A large interindividual variation between subjects was observed as the absorption of tocotrienols is dependent on food matrix and gut lipolysis. The accuracies of lower and upper limit of quantification ranged between $92 \%$ and $109 \%$ for intraday assays and $90 \%$ and $112 \%$ for interday assays. This method was successfully applied to quantify the total amount of four tocotrienol homologues in human plasma.
\end{abstract}

\section{Introduction}

Natural occurring vitamin E, or chemically known as tocochromanols, comprises of eight molecules having a 16carbon chain attached to a chromanol ring. Among them, tocopherols (TP) have saturated carbon side chain whereas tocotrienols (T3) contain three transdouble bonds in their carbon tails. Homologues of TP and T3, namely, $\alpha-, \beta-, \gamma-$, and $\delta$-homologues, are categorized based on the number and position of methyl groups on the chromanol ring $[1,2]$. The unsaturated carbon side chains present in tocotrienols give rise to their unique biological functions. In addition to their antioxidant properties, T3 have been widely investigated for their anticancer and neuroprotection as well as cholesterol lowering effects $[3,4]$. The distinctive properties of T3 had gained much attention in the research community in recent years, leading to the emergence of numerous human clinical trials. There is a critical need for a validated and optimized method for accurate quantification of T3 in biological fluids, especially human plasma.

In 1997, the American Oil Chemists' Society (AOCS) published an official method for quantification of vitamin E using high performance liquid chromatography (HPLC), suitable for detection in vegetable oils and fats. However, the direct application of this method on biological samples is limited due to the interference from blood proteins and the lack of sensitivity to detect small quantity. In the AOCS method, $\alpha$-TP were used as reference as T3 standards were not available. $\mathrm{Ng}$ et al. [5] reported that when calibrated using $\alpha$ $\mathrm{TP}$ as standard, the concentrations of vitamin $\mathrm{E}$ homologues appeared to be overestimated. A calibrated method using individual T3 homologues as standards is strongly recommended for accurate measurement of T3 concentrations. In addition, fluorescence detector gives higher sensitivity 
compared to UV detector as reported in AOCS method, enabling accurate measurement of small quantity in blood samples.

Over the years, several methods were developed for the quantification of vitamin E using HPLC. Most of these methods aim to determine the concentration of TP and T3 in food samples, including hazelnuts [6], olive oils [7], palm oils [8], and cereals [9]. A method using reverse phaseHPLC was reported by Yap et al. [10], to detect three T3 homologues as well as $\alpha$-TP in human blood samples. Lee et al. [11] have developed a method that was able to quantify vitamins $\mathrm{A}$ and $\mathrm{E}$ and various diet-derived carotenoids in human plasma. However, the method involved two different analytical columns and detectors. On the other hand, Abuasal and his coworkers have reported a specific $\gamma$-T3 analysis that can be adapted in both human and rat plasma [12]. In this study, we developed and validated a normal-phase-HPLC system with well-separated chromatograms able to detect small quantity of T3 in human blood samples.

\section{Materials and Methods}

2.1. Chemicals and Reagents. A mixed tocotrienols-tocopherols complex 50\% oil suspension containing $11.51 \% \alpha$-tocopherol, $12.99 \% \alpha$-tocotrienol, 2.55\% $\beta$-tocotrienol, 19.48\% $\gamma$ tocotrienol, and $7.28 \% \delta$-tocotrienol was used as an external standard. Internal standard 2,2,5,7,8-pentamethyl-6chromanol (PMC) and sodium chloride were purchased from Sigma-Aldrich (Malaysia) Sdn. Bhd. N-Hexane, 1,4-dioxane, 2-propanol, and ethanol (LiChrosolv grade) were purchased from Merck Sdn. Bhd. (Malaysia). Blank human plasma was obtained from Transfusion Medicine, University Malaya Medical Centre, Malaysia.

2.2. Instrumentation. $\mathrm{TP}$ and $\mathrm{T} 3$ homologues were analysed using Agilent 1100 series HPLC system equipped with quaternary pump (G1311A, serial number DE91609486), fluorescence detector (G1321A, serial number DE92002049), degasser (G1322A, serial number JP73021896), autosampler (G1313A, serial number DE91611414), and ChemStation Software. Chromatographic separations were performed using Luna $5 \mathrm{u}$ Silica $100 \mathrm{~A}$ ODS $(250 \times 4.60 \mathrm{~mm} \cdot \mathrm{d}, 5 \mu \mathrm{m}$ particle size) Hypersil column (Phenomenex, USA). Column was maintained at a pressure of $24 \pm 1$ bar and a temperature of $26^{\circ} \mathrm{C}$. Samples were injected at a volume of $100 \mu \mathrm{L}$ with flow rate of $1 \mathrm{~mL} / \mathrm{min}$ and a total run time of 30 minutes. Mobile phase was prepared using n-Hexane, 1,4-dioxane, and 2-propanol at $97.5: 2.0: 0.5 \% \mathrm{v} / \mathrm{v} / \mathrm{v}$, degassed by sonication prior to use. Vitamin E homologues were detected using fluorescence detector at excitation wavelength $295 \mathrm{~nm}$ and emission wavelength $325 \mathrm{~nm}$ (PMT-Gain at 10).

2.3. Preparation of Standard Solutions. Tocotrienols-tocopherols complex $50 \%$ oil suspension standard was prepared in a stock solution at $1000 \mathrm{ppm}$ in ethanol. Working standard solution of $100 \mathrm{ppm}$ was prepared by diluting stock standard solution in human plasma. Six dilutions ranging from 100, $75,50,10$, and 5 to $1 \mathrm{ppm}$ were prepared in human plasma.
PMC as internal standard (IS) was prepared in mobile phase at $2000 \mathrm{ppm}$ as a stock solution. Working standard solutions (10 ppm) were prepared by serial dilution in mobile phase.

2.4. Preparation of Human Plasma Samples. Human plasma samples were prepared according to method previously reported by Nesaretnam et al. [13] with slight modification. A volume of $0.5 \mathrm{~mL}$ of human plasma was added into a $12 \mathrm{~mL}$ glass test tube before spiking with $0.05 \mathrm{~mL}$ of $10 \mathrm{ppm}$ IS. The mixture was vortexed for 10 seconds and centrifuged at $2500 \mathrm{rpm}$ for 2 minutes at $4^{\circ} \mathrm{C}$. One $\mathrm{mL}$ of $0.9 \%$ sodium chloride $(\mathrm{NaCl})$ was added and vortexed vigorously, followed by an addition of $1 \mathrm{~mL}$ ethanol. The mixture was vortexed. Then, $5 \mathrm{~mL}$ of $\mathrm{n}$-hexane was added and the mixture was shaken for one hour at $1400 \mathrm{rpm}$ by using a mini shaker (IKA-VIBRAX-VXR, IKA, Germany). The mixture was centrifuged at $2500 \mathrm{rpm}$ for 15 minutes with a temperature of $4^{\circ} \mathrm{C}$, after which the upper organic layer was extracted and evaporated to dryness with nitrogen gas. Dried samples were reconstituted with $0.5 \mathrm{~mL}$ of mobile phase prior to analysis. Samples were analysed at $n=1$ and average plasma levels of tocotrienol homologues from 3 volunteers were determined.

2.5. Detection Range. Calibration curves were constructed using six standard concentrations of total tocotrienols ranging from 1 to $100 \mathrm{ppm}$. Peak areas of $\alpha$-tocotrienol, $\gamma$ tocotrienol, $\delta$-tocotrienol, and IS were plotted against their nominal concentrations and calibration curves were constructed based on linear equation $y=m x+c$, where $y$ represents the peak area, $x$ represents the nominal concentration, $m$ represents the slope, and $c$ represents the $y$-intercept values. Linear regression was evaluated using correlation coefficient $\left(R^{2}\right)$. Selectivity and specificity were determined with analysis of blank plasma. Limit of detection (LOD) was determined using lowest concentration with peak area of signal-to-noise $(\mathrm{S} / \mathrm{N})$ ratio of $\geq 3$. Limit of quantification (LOQ) was referred to as the lowest concentration on calibration curve at which quantitative results can be reported with a high degree of confidence that produced a peak with $\mathrm{S} / \mathrm{N}$ ratio of $\geq 10$.

2.6. Precision and Accuracy. Precision and accuracy of the method were evaluated by analysing the lower limit of quantification (LLOQ) and upper limit of quantification (ULOQ). Three replicates of LLOQ and ULOQ were analysed within the same day for intraday (within-day) precision. Interday (between-day) precision was obtained by analysing three replicates of LLOQ and ULOQ on consecutive days. Intraand interday precision were expressed as the percentage of relative standard deviation (\%RSD). Deviation from the true value was determined by comparing the obtained concentration with nominal concentration for intraday and interday accuracy and expressed as \% accuracy.

2.7. Supplementation of Tocotrienols. Subjects with metabolic syndrome were recruited from a Malaysian population with age category ranging from 25 to 56 years. Ethical approval 


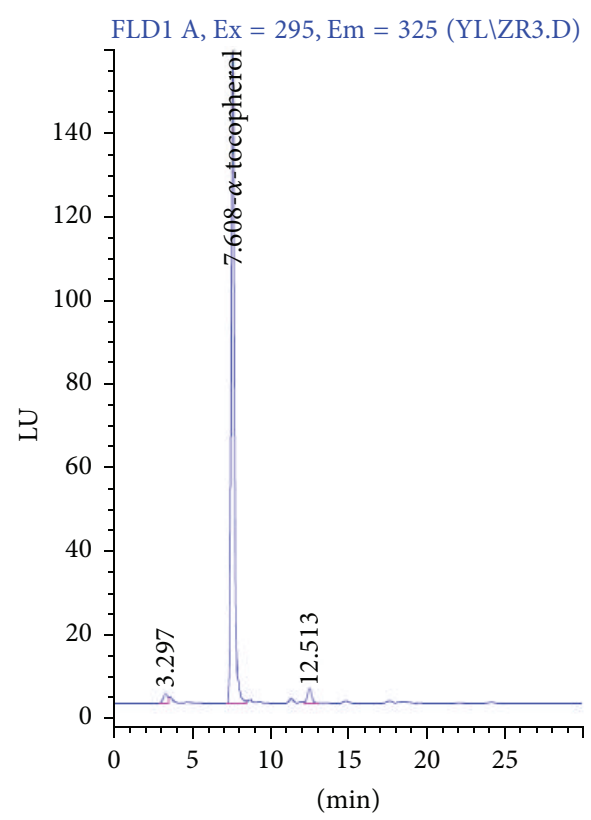

(a)

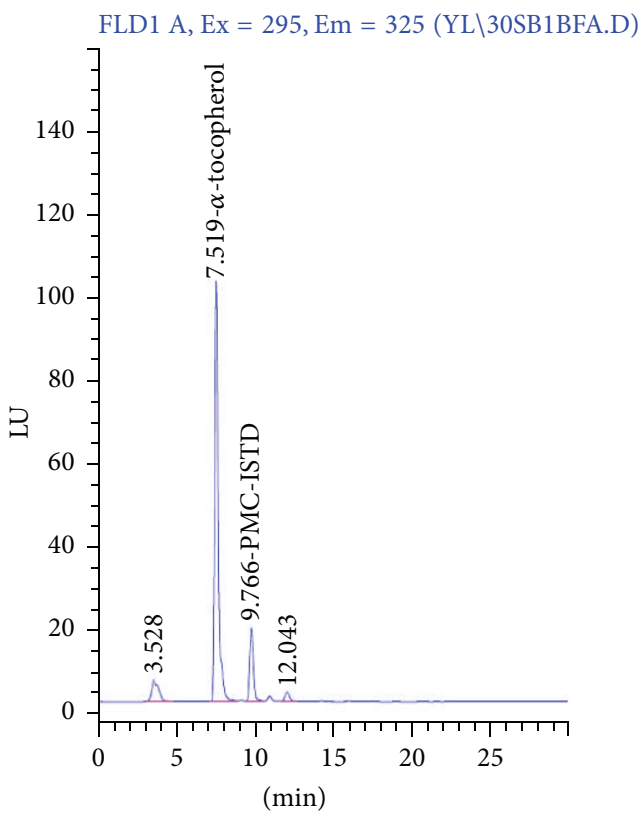

(b)

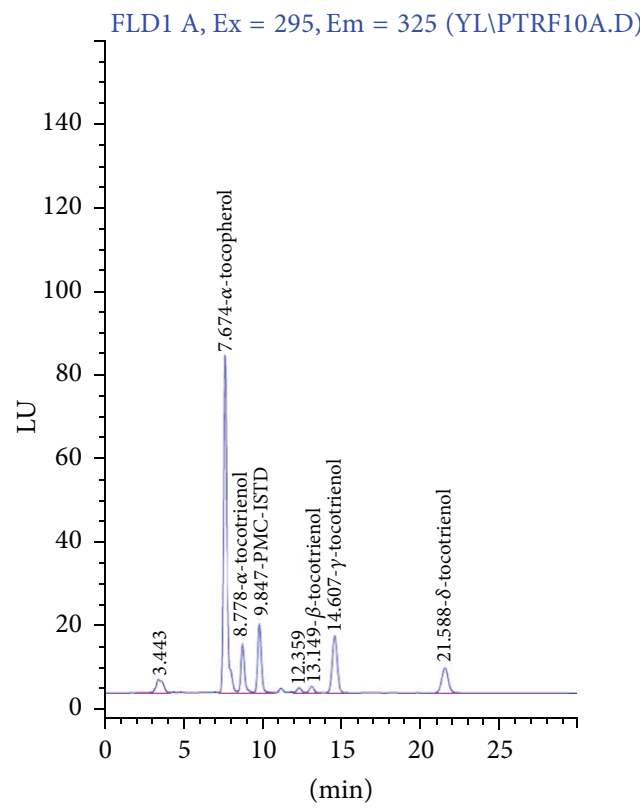

(c)

FIGURE 1: Chromatograms of (a) blank plasma, (b) plasma spiked with IS, and (c) plasma spiked with IS and vitamin E standard.

was obtained from the ethical committee from the University Putra Malaysia and registered at https://clinicaltrials .gov/ (NCT01631838). Subjects were supplemented with tocotrienol (Tocovid Suprabio) $200 \mathrm{mg}$ twice daily for 14 days. Blood samples were collected into K3EDTA vacutainer 4 hours after consumption of capsules on day 14. Plasma samples were collected using centrifugation at $3000 \mathrm{rpm}$ for 14 minutes at $4^{\circ} \mathrm{C}$. All human plasma samples were snapfrozen in liquid nitrogen and stored in $-80^{\circ} \mathrm{C}$ until analysis. The study was conducted according to Good Clinical Practice and Declaration of Helsinki guidelines 2008.

\section{Results and Discussion}

3.1. Selectivity and Specificity. Figure 1 illustrates the chromatograms of blank plasma and plasma spiked with IS and mixed tocopherol/tocotrienol standard. From the chromatogram of blank plasma, selectivity was verified as no interference peak was observed except for endogenous $\alpha$-TP and an unknown peak, which was detected at 7.9 and 12.5 minutes, respectively. The long elimination half-life of $\alpha$-TP, with reported value of around $20 \mathrm{~h}$, is the main reason for the presence of $\alpha$-TP peak in blank plasma $[14,15]$. This is similar 
TABLE 1: Detection range and linear correlation of T3 homologues.

\begin{tabular}{|c|c|c|c|c|}
\hline T3 homologues & Calibration range (ppm) & Slope $(m)$ & $Y$-intercept $(c)$ & $R^{2}$ \\
\hline$\alpha-\mathrm{T} 3$ & $0.1299-12.9900$ & 144.5622 & 5.8068 & 0.9994 \\
\hline$\beta-\mathrm{T} 3$ & $0.0255-2.5500$ & 121.2506 & 1.4434 & 0.9995 \\
\hline$\gamma$-T3 & $0.1948-19.4800$ & 150.5562 & 3.1899 & 0.9997 \\
\hline$\delta$-T3 & $0.0728-7.2800$ & 232.6348 & 2.0644 & 0.9997 \\
\hline
\end{tabular}

to the results reported by several clinical studies $[10,16,17]$. As such, the current method is not ideal to quantify $\alpha$-TP in plasma. Future efforts for calibration using synthetic plasma [18] or plasma stripped off endogenous compounds are warranted. Specificity of IS and T3 homologues was determined in Figures 1(b) and 1(c). Retention time of IS was observed at 9.8 minutes, in the absence of endogenous interference. The elution of T3 homologues followed the order of $\alpha$-T3, $\beta$-T3, $\gamma$-T3, and $\delta$-T3 at $8.8,13.1,14.6$, and 21.6 minutes, respectively. Although all peaks were generally well separated, the retention time for $\beta$-T3 and unknown peak in the plasma were relatively close. Identification of the unknown peak is warranted in future studies. Peak resolution of $\alpha$-TP can be improved as tailing effect was observed. According to $\mathrm{Ng}$ and Yuen May [19], a derivative of tocopherols, tocomonoenol, was eluted between $\alpha$-TP and $\alpha$-T3. The peak of tocomonoenol might be resolved with slight modification in the chromatographic separation of the current method to avoid overestimation of $\alpha$-TP. Nevertheless, limited availability of tocomonoenol standard might hamper the identification process.

3.2. Detection Range. Calibration curves were constructed using six standard concentrations ranging from 1 to $100 \mathrm{ppm}$ for total tocotrienols. For individual tocotrienol homologues, the detection range $(0.02 \mathrm{ppm}$ to $20 \mathrm{ppm})$ was summarized in Table 1. Variations in peak area for IS were relatively small (ranges between 5-8 LU) within-day and between-day. As such, calibration curves were constructed based on peak area against nominal concentrations of T3. However, it is reasonable to construct calibration curves using the peak area ratios of T3 homologues against IS in future work in order to eliminate the variations in peak area. In this method, the correlation coefficients $\left(R^{2}\right)$ for all T3 homologues were higher than 0.999, which were sufficiently high to indicate a linear correlation between the peak area and T3 concentrations. On the other hand, each T3 homologue varied in their slope and $y$-intercept values (Table 1). Our results correlated with recommendations by $\mathrm{Ng}$ and Yuen May [19] that quantification of vitamin E homologue should be correlated with their own standards to avoid over- or underestimation. The LLOQ and ULOQ of total tocotrienols were determined at $1 \mathrm{ppm}$ and $100 \mathrm{ppm}$ for total tocotrienols, respectively. For individual homologues, the LLOQ and ULOQ correlate with the calibration range summarized in Table 1 . Table 2 summarizes the LOD and LOQ of T3 homologues in human plasma. Chromatogram of LOD/LOQ was presented in Supplementary Data (in Supplementary Material available online at http://dx.doi.org/10.1155/2015/357609). All T3 homologues
TABLE 2: LOD and LOQ of T3 homologues.

\begin{tabular}{lcc}
\hline T3 homologues & LOD $(\mathrm{ppm})$ & LOQ $(\mathrm{ppm})$ \\
\hline$\alpha$-T3 & 0.1299 & 0.1299 \\
$\beta$-T3 & 0.0255 & 0.0255 \\
$\gamma$-T3 & 0.1948 & 0.1948 \\
$\delta$-T3 & 0.0728 & 0.0728 \\
\hline
\end{tabular}

TABLE 3: Precision and accuracy of LLOQ and ULOQ.

\begin{tabular}{lcc}
\hline & Precision (\%RSD) & Accuracy (\% accuracy) \\
\hline Intraday assay & & \\
LLOQ & 3.97 & 109.65 \\
ULOQ & 0.34 & 92.87 \\
Interday assay & & \\
LLOQ & 2.00 & 112.81 \\
ULOQ & 2.13 & 90.11 \\
\hline
\end{tabular}

appeared to have similar LOD and LOQ as dilutions below the LOQ did not yield S/N ratio of $\geq 3$. The LOD and LOQ observed ranged from $0.0255 \mathrm{ppm}$ in $\beta$-T3 to $0.1948 \mathrm{ppm}$ in $\gamma$-T3, higher values than that reported by Yap et al. [10], Lee et al. [11], and Abuasal et al. [12]. Nevertheless, $\mathrm{S} / \mathrm{N}$ ratio of $\beta$ T3 was relatively low compared to the other T3 homologues due to its low concentration in the tocotrienols-tocopherols complex 50\% standard. Using pure standards of individual T3 homologues will significantly improve the accuracy of T3 quantification in future work.

3.3. Precision and Accuracy. Precision of the method was determined by quantifying the \%RSD of LLOQ and ULOQ in intra- and interday analysis (Table 3 ). The \%RSD was found to be less than 5\% for both LLOQ and ULUQ at intra- and interday analysis. The variation is marginally higher than $2 \%$ as recommended by the FDA Guidance for Industry on Bioanalytical Method Validation for bioequivalence. The inconsistency in injection volume and detector might give rise to the variation, especially for low concentrations in LLOQ. The precision of this method can be improved by calibrating using area ratio of analyte peak to internal standard peak. The area ratio calibration is more reproducible and eliminates the instrumentation inconsistency [20]. Accuracy of LLOQ and ULOQ ranged between 92 and 109\% for intraday assays and 90 and $112 \%$ for interday assays. The International Conference on Harmonisation of Technical Requirements for Registration of Pharmaceuticals for Human Use (ICH) guidelines suggested \% accuracy within 80-120\% for reliable estimation of the true values. 


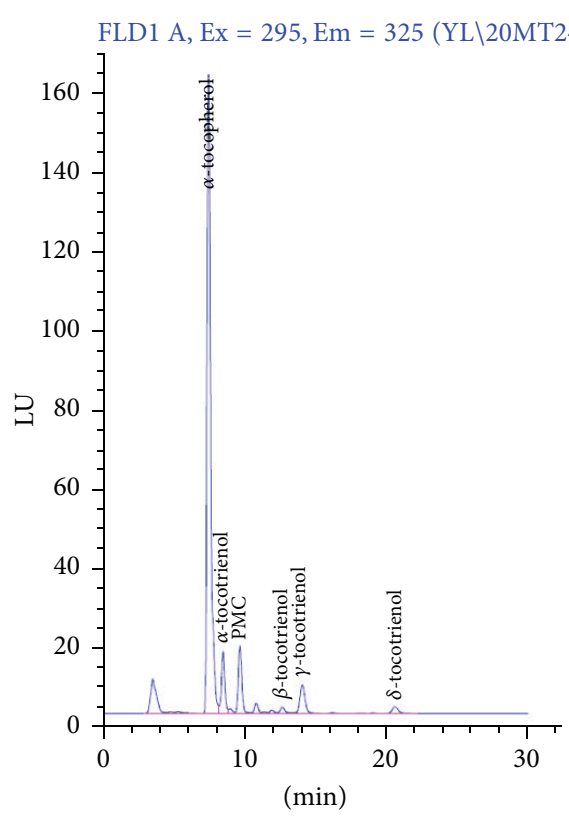

(a)

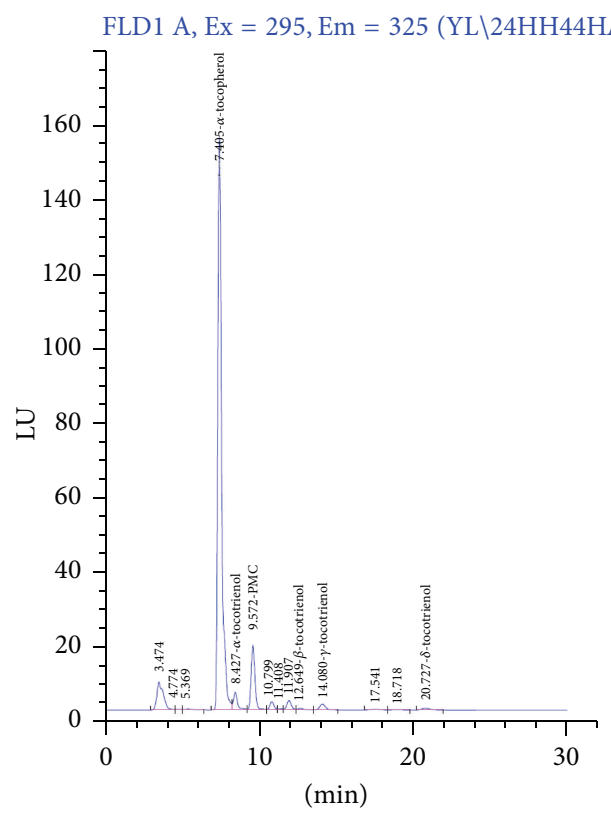

(b)

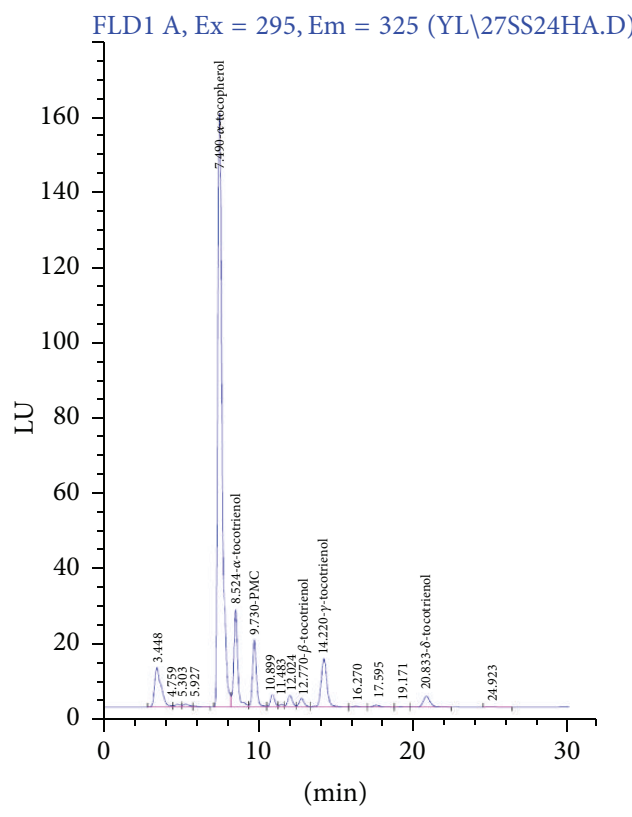

(c)

FIGURE 2: Chromatograms for human plasma of three male volunteers $(a-c)$ after consumption of $200 \mathrm{mg}$ mixed tocotrienols.

3.4. Concentration of Tocotrienols in Human Plasma Samples. Figure 2 illustrates the chromatograms for human plasma of three male volunteers after consumption of $200 \mathrm{mg}$ mixed tocotrienols. At 4 hours after supplementation, the total amount of $\mathrm{T} 3$ detected in plasma ranged from $0.93 \mathrm{ppm}$ to 6.13 ppm. According to Yap et al. [10] and Fairus et al. [21], highest plasma concentration of T3 $\left(T_{\max }\right)$ was reached at 4-5 hours, dependent on the different homologues. A large interindividual variation between subjects was observed. This phenomenon was previously reported by Heng et al. [22], as tocotrienols were absorbed via the lipoprotein pathway, which is dependent on food matrix and gut lipolysis [23]. Table 4 summarizes the levels of different T3 homologues detected. Alpha-T3 and $\gamma$-T3 appeared to be most abundant, correlating with their high amount in Tocovid Suprabio capsules (61.52 mg $\alpha$-T3, $112.80 \mathrm{mg} \gamma$-T3, and $25.68 \mathrm{mg} \delta$-T3). The recovery of IS was calculated at $100 \%, 119 \%$, and $128 \%$, respectively, for subjects $\mathrm{A}, \mathrm{B}$, and $\mathrm{C}$. Three unidentified peaks were detected at approximately $10.8,11.9$, and 17.6 minutes. They were postulated as $\beta$-, $\gamma$-, and $\delta$-tocopherols although further identification is needed with respective standards. 
TABLE 4: Average concentrations of T3 homologues detected in human plasma of 3 male volunteers.

\begin{tabular}{lccccc}
\hline $\begin{array}{l}\text { T3 } \\
\text { homologues }\end{array}$ & $\begin{array}{c}\text { Subject A } \\
(\mathrm{ppm})\end{array}$ & $\begin{array}{c}\text { Subject B } \\
(\mathrm{ppm})\end{array}$ & $\begin{array}{c}\text { Subject C } \\
(\mathrm{ppm})\end{array}$ & $\begin{array}{c}\text { Mean } \\
(\mathrm{ppm})\end{array}$ & $\begin{array}{c}\mathrm{SD} \\
(\mathrm{ppm})\end{array}$ \\
\hline$\alpha$-T3 & 1.7423 & 0.5305 & 3.0948 & 1.7892 & 1.283 \\
$\beta$-T3 & 0.3059 & 0.0877 & 0.4383 & 0.2773 & 0.177 \\
$\gamma$-T3 & 1.2006 & 0.2521 & 2.2102 & 1.2210 & 0.979 \\
$\delta$-T3 & 0.2221 & 0.0631 & 0.3893 & 0.2248 & 0.163 \\
\hline
\end{tabular}

\section{Conclusion}

A simple, specific, and reproducible HPLC method was developed and validated for detection of tocotrienols in human plasma. The method is selective and specific for detection of $\alpha_{-}^{-}, \beta_{-}, \gamma^{-}$, and $\delta$-T3 in human plasma. Validation of the method showed sufficient precision and accuracy at a concentration ranging from 0.02 to $20 \mathrm{ppm}$. Stability tests upon freeze-thaw cycles and ambient temperatures are warranted to verify the conditions of analysis and sample storage. The current method has been applied for quantification of tocotrienol levels in plasma of human volunteers consuming mixed tocotrienols. This method will be useful for clinical studies for accurate quantification of tocotrienols in biological fluids, especially human plasma.

\section{Conflict of Interests}

The authors declare that there is no conflict of interests regarding the publication of this paper.

\section{Acknowledgments}

This project is funded by the Malaysian Palm Oil Board (MPOB). The authors thank the Director-General of MPOB for permission to publish this paper. Hui-Ling Che, Doryn Meam-Yee Tan, and Yee-Lin Gan are funded under the MPOB Graduate Students Assistantship Scheme for their postgraduate studies.

\section{References}

[1] C. K. Sen, S. Khanna, and S. Roy, "Tocotrienols: vitamin E beyond tocopherols," Life Sciences, vol. 78, no. 18, pp. 20882098, 2006.

[2] K. Nesaretnam, W. W. Yew, and M. B. Wahid, "Tocotrienols and cancer: beyond antioxidant activity," European Journal of Lipid Science and Technology, vol. 109, no. 4, pp. 445-452, 2007.

[3] B. B. Aggarwal, C. Sundaram, S. Prasad, and R. Kannappan, "Tocotrienols, the vitamin E of the 21st century: its potential against cancer and other chronic diseases," Biochemical Pharmacology, vol. 80, no. 11, pp. 1613-1631, 2010.

[4] K. Nesaretnam, "Multitargeted therapy of cancer by tocotrienols," Cancer Letters, vol. 269, no. 2, pp. 388-395, 2008.

[5] M. H. Ng, Y. M. Choo, A. N. Ma, C. H. Chuah, and H. M. Ali, "Separation of vitamin E (tocopherol, tocotrienol, and tocomonoenol) in palm oil," Lipids, vol. 39, no. 10, pp. 1031-1035, 2004 .
[6] J. S. Amaral, S. Casal, D. Torres, R. M. Seabra, and B. P. P. Oliveira, "Simultaneous determination of tocopherols and tocotrienols in hazelnuts by a normal phase liquid chromatographic method," Analytical Sciences, vol. 21, no. 12, pp. 15451548, 2005.

[7] S. C. Cunha, J. S. Amaral, J. O. Fernandes, and M. B. P. P. Oliveira, "Quantification of tocopherols and tocotrienols in Portuguese olive oils using HPLC with three different detection systems," Journal of Agricultural and Food Chemistry, vol. 54, no. 9, pp. 3351-3356, 2006.

[8] S. Ong, C. H. Xu, V. Teo et al., "Comprehensive quantification of palm vitamin $\mathrm{E}$ by normal phase high performance liquid chromatography," Palm Oil Developments, vol. 52, pp. 11-17, 2010.

[9] M. N. Irakli, V. F. Samanidou, and I. N. Papadoyannis, "Optimization and validation of the reversed-phase highperformance liquid chromatography with fluorescence detection method for the separation of tocopherol and tocotrienol isomers in cereals, employing a novel sorbent material," Journal of Agricultural and Food Chemistry, vol. 60, no. 9, pp. 20762082, 2012.

[10] S. P. Yap, T. Julianto, J. W. Wong, and K. H. Yuen, "Simple high-performance liquid chromatographic method for the determination of tocotrienols in human plasma," Journal of Chromatography B: Biomedical Sciences and Applications, vol. 735, no. 2, pp. 279-283, 1999.

[11] B.-L. Lee, A.-L. New, and C.-N. Ong, "Simultaneous determination of tocotrienols, tocopherols, retinol, and major carotenoids in human plasma," Clinical Chemistry, vol. 49, no. 12, pp. 20562066, 2003.

[12] B. Abuasal, S. Thomas, P. W. Sylvester, and A. Kaddoumi, "Development and validation of a reversed-phase HPLC method for the determination of $\gamma$-tocotrienol in rat and human plasma," Biomedical Chromatography, vol. 25, no. 5, pp. 621-627, 2011.

[13] K. Nesaretnam, K. R. Selvaduray, G. Abdul Razak, S. D. Veerasenan, and P. A. Gomez, "Effectiveness of tocotrienolrich fraction combined with tamoxifen in the management of women with early breast cancer: a pilot clinical trial," Breast Cancer Research, vol. 12, no. 5, article R81, 2010.

[14] N. E. Bateman and D. A. Uccellini, "Kinetics of D- $\alpha$-tocopherol in a water soluble base in man," Journal of Pharmacy and Pharmacology, vol. 37, no. 10, pp. 728-729, 1985.

[15] T. Julianto, K. H. Yuen, and A. Mohammad Noor, "Improved bioavailability of vitamin E with a self emulsifying formulation," International Journal of Pharmaceutics, vol. 200, no. 1, pp. 53-57, 2000.

[16] R. P. Mensink, A. C. van Houwelingen, D. Kromhout, and G. Hornstra, "A vitamin E concentrate rich in tocotrienols had no effect on serum lipids, lipoproteins, or platelet function in men with mildly elevated serum lipid concentrations," The American Journal of Clinical Nutrition, vol. 69, no. 2, pp. 213-219, 1999.

[17] K. H. Yuen, J. W. Wong, A. B. Lim, B. H. Ng, and W. P. Choy, "Effect of mixed-tocotrienols in hypercholesterolemic subjects," Functional Food in Health and Disease, vol. 3, pp. 106-117, 2011.

[18] S. N. Alvi and M. M. Hammami, "Validated HPLC method for determination of caffeine level in human plasma using synthetic plasma: application to bioavailability studies," Journal of Chromatographic Science, vol. 49, no. 4, pp. 292-296, 2011.

[19] M. H. Ng and C. Yuen May, "Chromatographic analyses of tocopherols and tocotrienols in palm oil," Journal of Chromatographic Science, vol. 50, no. 3, pp. 283-286, 2012. 
[20] S. Hansen, S. Pedersen-Bjergaard, and K. Rasmussen, "Quantification and quality of analytical data," in Introduction to Pharmaceutical Chemical Analysis, John Wiley \& Sons, New York, NY, USA, 2011.

[21] S. Fairus, R. M. Nor, H. M. Cheng, and K. Sundram, "Postprandial metabolic fate of tocotrienol-rich vitamin $\mathrm{E}$ differs significantly from that of $\alpha$-tocopherol," The American Journal of Clinical Nutrition, vol. 84, no. 4, pp. 835-842, 2006.

[22] E. C. Heng, S. A. Karsani, M. A. Rahman, N. A. A. Hamid, Z. Hamid, and W. Z. W. Ngah, "Supplementation with tocotrienolrich fraction alters the plasma levels of Apolipoprotein A-I precursor, Apolipoprotein e precursor, and C-reactive protein precursor from young and old individuals," European Journal of Nutrition, vol. 52, no. 7, pp. 1811-1820, 2013.

[23] J.-Y. Fu, H.-L. Che, D. M.-Y. Tan, and K.-T. Teng, "Bioavailability of tocotrienols: evidence in human studies," Nutrition \& Metabolism, vol. 11, no. 1, article 5, 2014. 

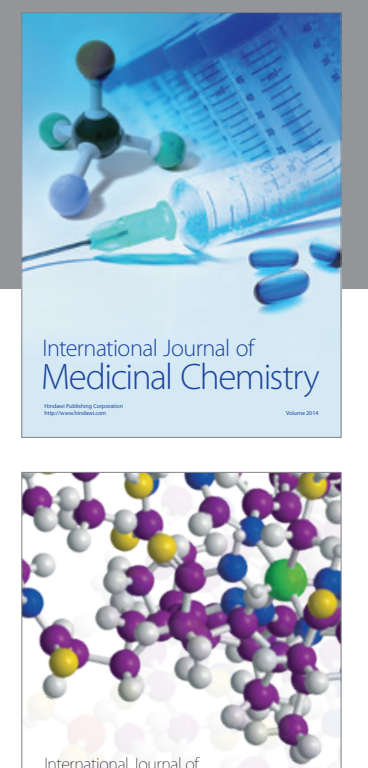

\section{Carbohydrate} Chemistry

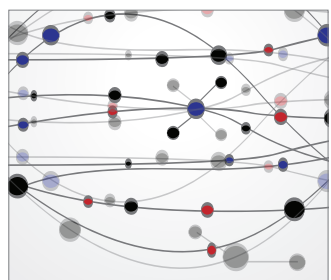

The Scientific World Journal
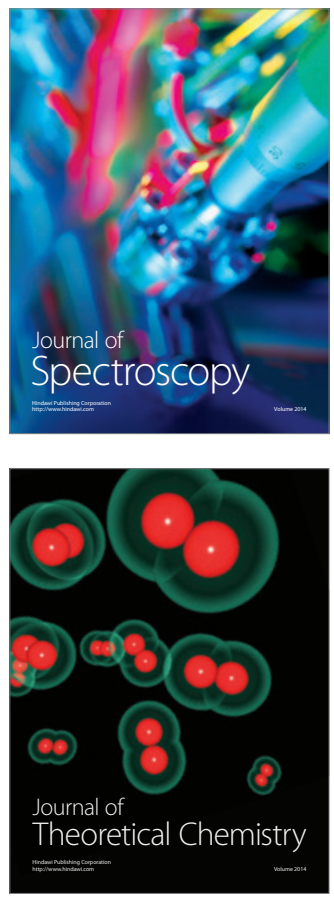
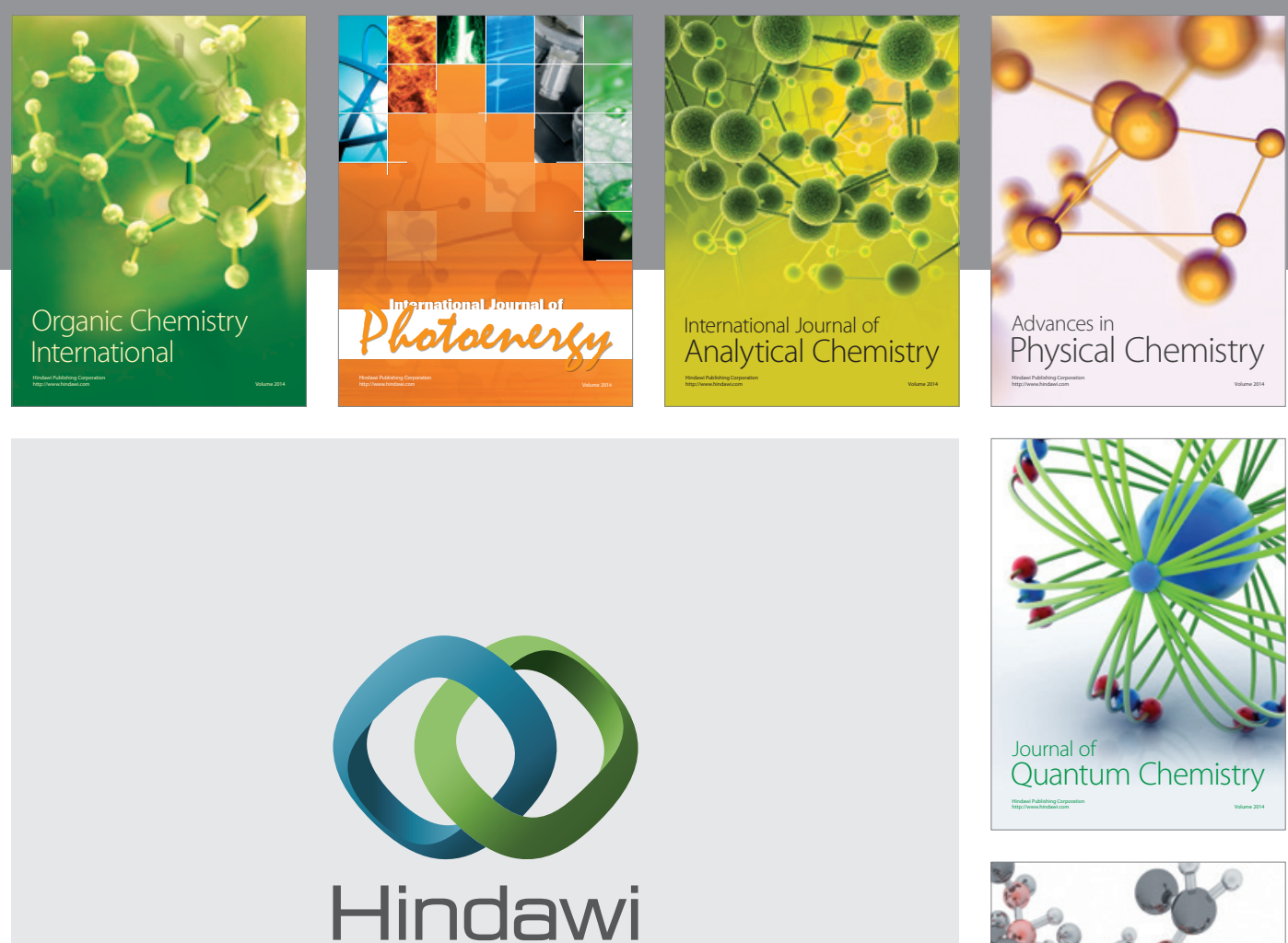

Submit your manuscripts at

http://www.hindawi.com

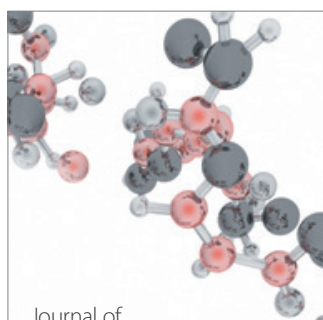

Analytical Methods

in Chemistry

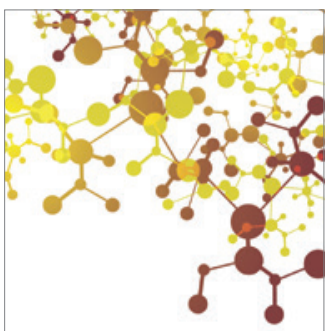

Journal of

Applied Chemistry

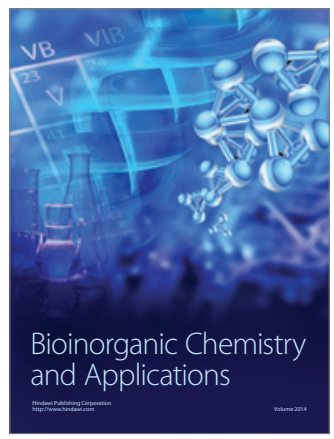

Inorganic Chemistry
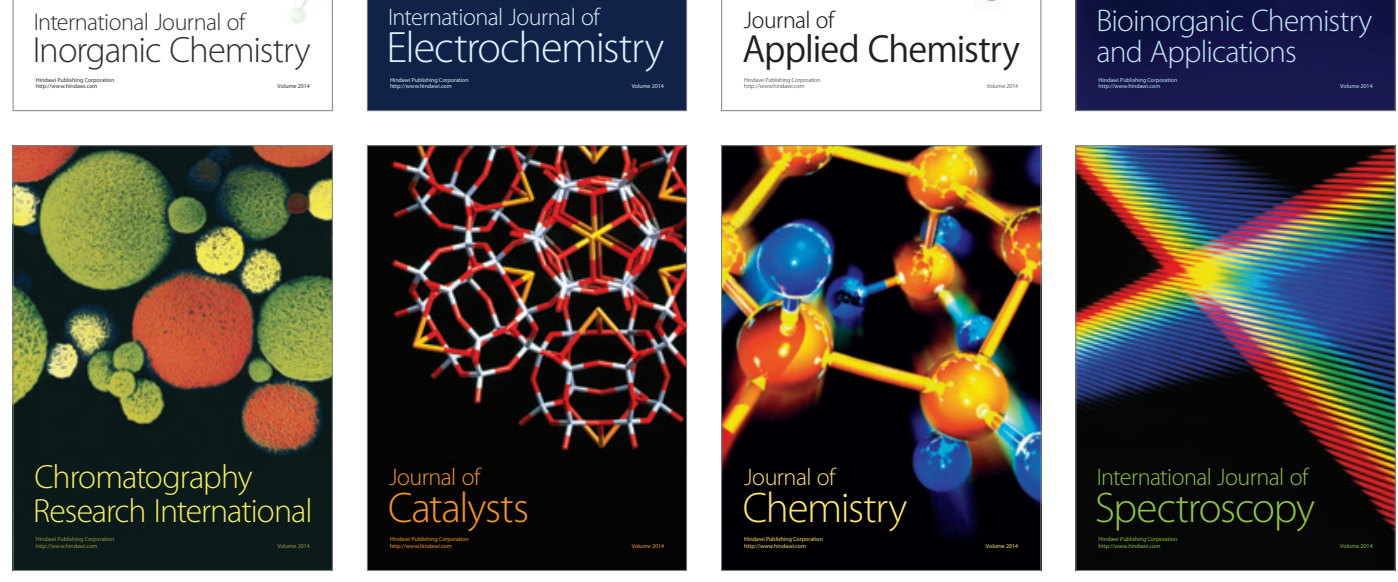